\title{
La latinización de los Estados Unidos: Inmigración, cultura y transformación
}

\author{
- Pedro Castillo \\ Universidad de California en Santa Cruz
}

\section{Introducción}

La aventura y el intento por establecerse en un lugar que ofrezca mejores oportunidades es la historia de Estados Unidos, desde la colonización hasta el presente. La economía y la cultura de Estados Unidos, su propio sentido como nación, han sido delineadas por las migraciones hacia ese país y por los movimientos poblacionales suscitados dentro del mismo. Irlandeses, italianos, judíos rusos y otros europeos llegan a la costa occidental a finales del siglo XIX y principios del XX y pronto se extienden por todo el país. De este modo, los colonizadores escandinavos delimitan granjas en las llanuras, los mormones se refugian en Utah, los afroamericanos dejan el sur para dirigirse hacia las ciudades del norte y del medio oeste y así ha continuado.

El capítulo actual de esta épica interminable describe la migración de los latinos a Estados Unidos y la movilidad geográfica interna hacia el medio oeste y el sur. Como siempre, los recién llegados buscan una vida mejor. Como siempre, están deseosos de trabajar bajo condiciones inadmisibles para la mayoría de los residentes. Los inmigrantes y residentes latinos provocan cambios en las comunidades que no siempre gustan a los residentes que llevan más tiempo. Como siempre, su nueva presencia en la ciudad se enfrenta a una mezcla de bienvenida y resistencia. Esto surge de un sentido de desplaza-

La latinización de los Estados Unidos: Inmigración, cultura y transtormación 
miento que puede ser muy marcado en la gente que siempre ha habitado en el mismo lugar. De repente, el pueblo o ciudad se viste de otra manera, toca otra música, consume comida diferente, habla un idioma distinto.

Nuevas caras, nuevas ideas, nuevos estilos de vida y nuevos reçursos penetran en un orden social previamente establecido. Se entrelazan, se mezclan, se desdibujan, se absorben. Los personajes son otros, pero el sorprendente cambio social provocado por la inmigración sigue siendo uno de los temas constantes de la saga estadounidense.

\section{La población de los Estados Unidos nacida en extranjero}

Según datos presentados en marzo de 1997, el $9.7 \%$ de la población de los Estados Unidos había nacido en extranjero. Éste es el porcentaje más alto de inmigrantes nacidos fuera del país desde la Gran Depresión de 1930 y se ha incrementado a partir de 1970 , cuando el $4.8 \%$ de su población había nacido en el extranjero. Durante este siglo, el porcentaje experimentó un decremento que va del $14.7 \%$ en 1910 , al $4.8 \%$ en 1970 . Pero a partir de entonces, definitivamente se ha incrementado. La población nacida en el extranjero entre 1870 y 1920 es el resultado de la inmigración europea. A pesar de que ésta se elevó de manera drástica durante los últimos veinticinco años, continuó por debajo del nivel existente a principios de este siglo, cuando arribó una fuerte ola de europeos del este y los inmigrantes se convirtieron en casi el $15 \%$ de la población total.

En marzo de 1997, aproximadamente 25.8 millones de residentes de los Estados Unidos habían nacido fuera. California encabeza la lista con $24.9 \%$, le siguen Nueva York (19.8\%), Florida (16.4\%), Nueva Jersey (15.4\%) y Texas (11.3\%). El $51 \%$ del total de la población nacida en el extranjero (13.1 millones) estaba compuesto por nacidos en América Central, Sudamérica y el Caribe. Cerca del $27 \%$ de los nacidos en el extranjero eran originarios de Asia y $17 \%$ provenía de Europa. De los latinos, el $27 \%$ había nacido en México ( 7 millones). De la población nacida en el extranjero, cerca del 35 $\%$ lo constituían ciudadanos naturalizados, en tanto que casi el $65 \%$ no tenía la ciudadanía (Census Bureau, EE.UU., 1997. La población nacida en el extranjero en los Estados Unidos: Marzo 1997 (actualización). Para ver informes actuales de población y características de la misma, cfr. pp. 20-507).

\subsection{Incremento del número de latinos en los Estdos Unidos}

Alimentado por la inmigración y un mayor número de nacimientos, el número de latinos que vive en los Estados Unidos creció a casi 30 millones 
en 1997; se proyecta que esta tendencia se triplique hacia el 2050, según la Oficina de Censos. Se prevé que la población latina se duplique entre 1980 y el 2005 . Hoy en día, los latinos conforman el $11 \%$ de la población de los Estados Unidos, superando el $9 \%$ de 1990. Hacia el 2005, el número de residentes latinos ascenderá a más de 36 millones en comparación con alrededor de 35.5 millones de afroamericanos. Será el grupo minoritario más grande del país. La Oficina de Censos estima que ese número alcanzará el $25 \%$ hacia el 2050, cuando se espera que la población latina alcance los 96.5 millones — más de diez veces su número en 1970—. La inmigración proveniente de México y de Centroamérica es la causa principal del alto incremento de latinos en los Estados Unidos. Este cambio de siglo es completamente diferente del anterior, cuando la gran mayoría de inmigrantes provenía de Europa. En 1900, el 98 \% de los inmigrantes llegó de ese continente. Hacia 1990, el porcentaje de inmigrantes de Europa cayó al $26 \%$, en tanto que el porcentaje de inmigrantes de países hispanoparlantes alcanzó el $43 \%$ (Steven A. Holmes, Population Gap Narrows Between Latinos, Blacks. New York Times, 7 de agosto de 1998).

Según la información más reciente, menos de la mitad —es decir, el $44 \%$ - de la población latina actual nació fuera de los Estados Unidos. Debido a que esta población es más joven, tiende a tener más hijos. Aproximadamente un tercio, esto es, el $35.7 \%$ de los latinos, tenía dieciocho años o menos. Más aún, se cree que la población blanca con edad de hasta dieciocho años disminuirá entre 1990 y 2050. A principios de 1997, el número de escuelas para niños latinos fue por primera vez mayor que el número de escuelas para niños afroamericanos (Según informes del National Center For Health Statistics.) La Oficina de Censos también afirma que un número mayor de latinos permanece casados, en relación con la población total. Casi siete de cada diez familias latinas (68.2 \%) estaban conformadas por parejas casadas en 1997, mientras que para las familias blancas este número osciló alrededor del $60 \%$ para las afroamericanas fue de $42 \%$ y para las asiáticas, de $62 \%$. Parece ser que los latinos tienen predilección por la vida en familia.

La población de los Estados Unidos que no es blanca comienza a incrementarse, y la Oficina de Censos estima que, para el 2050, la población total se compondrá como sigue: $52.8 \%$ blanca, $24.5 \%$ latina, $13.6 \%$ afroamericana, $8.2 \%$ de origen asiático, y $0.9 \%$, india. Actualmente, la población de Estados Unidos es blanca en un $73.6 \%$; afroamericana, en un $12 \%$; latina, en un $10.2 \%$; de origen asiático, en un $3.3 \%$ e india en un 0.7 $\%$. Todavía hay más: la población del país se incrementará de 270 a 394 millones hacia el 2050. El índice de crecimiento actual es muy modesto,

La latinización de los Estodos Unidos: Inmigración, cultura y transformación 
menor al $1 \%$ por año, y es el más bajo desde la década de los treinta; se moverá aún más lento después del 2025. Estos patrones de crecimiento producirán un cambio dramático en la imagen étnica racial de Estados unidos. Incluso, sin tomar en cuenta la inmigración, la población latina continuará creciendo más rápido que la blanca, porque es más joven y tiene un índice más alto de fertilidad. Por otro lado, también está creciendo rápidamente la población integrada por blancos que tienen más de cincuenta años de edad. En el futuro, la mitad del mercado estadounidense estará integrada por personas de cincuenta o más años, en tanto la otra mitad se compondrá de latinos y asiáticos.

\section{La población latina}

El total de la población latina en los Estados Unidos es de 28,438,000 de personas. De estas, 18,039,000 son mexicanas; 3,123,000, son portorriqueños; 1,127,000, cubanos, mientras que 4,060,000 proceden de Centro y Sudamérica y 2,089,000 provienen de otros países latinos. El 63\% de la población latina es de origen mexicano; de ella, el $61.9 \%$ nació en los Estados Unidos y $36.8 \%$ nació fuera. El $56.8 \%$ de los portorriqueños nació en Estados Unidos, en tanto que el $39.1 \%$ nació en Puerto Rico. El 30.2 $\%$ de los cubanos nació en Estados Unidos, mientras que el 68.6 \% nació en Cuba. El $30.3 \%$ de los centro y sudamericanos nació en Estados Unidos, en contraste con el $67.6 \%$ que nació fuera de ese país. El $70.5 \%$ de quienes son originarios de otras naciones latinos nació en Estados Unidos, en tanto el $27.3 \%$ nació fuera de ese país.

\subsection{El caso de California}

En 1997, la población del estado estaba compuesta de la siguiente manera: El $52.5 \%$ eran blancos; $29.8 \%$, latinos; $10.7 \%$ eran de origen asiático y un $6.9 \%$, afroamericanos. Hacia el 2040 , se proyecta que haya un 49.7 $\%$ de latinos; que el $32.4 \%$ de la población sea blanca; mientras que el 11.8 $\%$ sean de origen asiático y el $5.9 \%$, afroamericanos. El condado de los Ángeles cuenta ahora con un $43.5 \%$ de latinos y se pronostica que en el 2040 este porcentaje llegará al $69.1 \%$ (Robert Rosenblatt, Latinos, Asian Lead Rise in US Population Los Angeles Times, 14 de marzo de 1996).

\section{2. ¿Por qué se da la migración mexicana a los Estados Unidos?}

Pocos lugares en los Estados Unidos parecerían menos atractivos para los mexicanos que Alaska o Hawai. Pero es en dichos estados donde miles de 
mexicanos han estado emigrando recientemente, cambiando así sus esquemas laborales.

Los mexicanos están trabajando en los cañaverales y en los plantíos de piña de Hawai, así como en la industria pesquera de Alaska. A través de las décadas, millones de mexicanos se han dirigido desde su país natal hacia el norte: a los campos de California y a los ranchos ganaderos de Texas; así como a laborar en la industria del medio oeste, especialmente en Chicago. Ahora, los trabajadores mexicanos están encontrando nuevas fronteras, en respuesta a la demanda de mano de obra barata en lugares de Estados Unidos muy alejados de su frontera sudoeste. Un mayor número de ellos, no solamente está viajando a Alaska y Hawai, sino también al cultivo de hongos en Pennsylvania, a la industria de la construcción en Atlanta, al ordeño de vacas en Idaho, a la cosecha de tabaco en Carolina del Norte, a la cría de cerdos en lowa, a las granjas avícolas de Carolina del Sur, o a los rastros de Dodge en Kansas. La dispersión de los mexicanos en los cincuenta estados de la Unión Americana se ha incrementado a partir de 1986, fecha en que fue aprobada la reforma de inmigración, así como el Control Act, legalizando, así, la situación de más de dos millones de mexicanos que habían estado trabajando en ese país sin documentos. La tendencia también se aceleró a partir de 1994, debido a la crisis del peso, cuando muchos mexicanos de clase media comenzaron a viajar hacia el norte en busca de trabajo. California había sido el principal destino de los mexicanos durante la Segunda Guerra Mundial, pero debido a que la economía de ese estado cayó en una recesión, y a que el fervor antiinmigrante se esparció, muchos mexicanos se han ido a otros lugares.

Por ejemplo, cientos de mexicanos inmigrantes se han establecido tanto en el centro como en los alrededores del poblado rural Turner, de cinco mil habitantes, en Maine. Trabajan para el mayor productor de huevo rojo en el mundo. Aunque los mexicanos comprenden menos del $1 \%$ de población en Maine, es un hecho que la presencia de este grupo poblacional está creciendo. Gloria González, quien llegó a Turner hace cinco años con su esposo y sus cuatro hijos, afirma que "siempre se extraña la casa —el idioma, la cultura, la comida...- pero vinimos a Maine por algo más: para construir un mejor futuro para nuestros hijos (Patrick McDonnell, Mexican Arrivals Seek New Frontiers. Los Angeles Times, $1^{\circ}$ de enero de 1998.)".

El éxodo mexicano que ha tenido lugar aproximadamente durante las dos últimas décadas, hoy en día representa la ola migratoria jamás vista de un grupo a los Estados Unidos, eclipsando por mucho las llegadas previas de irlandeses, italianos y judíos. Más de siete millones de mexicanos inmigrantes

Lo latinización de los Estodos Unidos: Inmigración, culturo y transtormación 
nacidos en México, ahora viven en Estados Unidos; la gran mayoría ha llegado a partir de 1970. El alto número, combinado con otros factores - la proximidad de los inmigrantes mexicanos a su país, la fuerte presencia de publicaciones en español, la radio y la televisión, la facilidad para cruzar la frontera y las vías de comunicación, en general — puede indicar una redefinición de la experiencia inmigrante estadounidense: el inmigrante transnacional, "las ciudades sin fronteras", "MexAmerica". Los mexicanos mantienen su "mexicanidad", incluso mientras se convierten en estadounidenses — de un modo que no pudieron los italianos, los irlandeses, los judíos, ni los griegos-. Esta migración mexicana y latina altera, profunda y sutilmente, la sociedad estadounidense. Muestras claras de ello son las señalizaciones en español y el predominio de música y comida latinas.

La evidencia sugiere que más y más mexicanos se establecen en los Estados Unidos permanentemente, a diferencia de los patrones de inmigración de ida y vuelta de las generaciones anteriores. Los mexicanos trabajan en industrias de temporada, desde hoteles y restaurantes, hasta la industria avícola y los procesadores de cárnicos. En un patrón de "migración en cadena", que se da en toda la Unión Americana, llegan a una comunidad algunos trabajadores mexicanos y les siguen otros. Las oportunidades de trabajo se dan a conocer a través de una eficiente red de comunicación en las familias y en la comunidad, que penetra profundamente en México.

Conforme un mayor número de mexicanos y mexicoamericanos llegan a pequeñas ciudades y pueblos de Estados Unidos, transforman el panorama cultural. Estos latinos son bien recibidos porque realizan trabajos poco calificados en la industria y contribuyen a revitalizar economías debilitadas. El movimiento más dramático es el éxodo de California. De 1990 a 1996, 500,000 latinos salieron de California hacia los estados rurales. El alto costo de la vida, el crimen urbano y el clima antiinmigrante en California provocaron que el estado dorado perdiera su resplandor. Durante décadas, California fue el destino más común para los mexicanos inmigrantes que abandonaban los estados rurales de Michoacán, Jalisco, Zacatecas y Guanajuato. Hoy en día, muchos trabajadores que viajan rumbo al norte van directamente a la zona decisiva. Los latinos se han vuelto indispensables en la industria empacadora avícola y ganadera, dicen los empresarios, porque las bajas tasas de desempleo han hecho muy difícil contratar trabajadores locales. La población latina está expandiéndose en los lugares menos pensados. De 1990 a 1996, esta población creció en un $104 \%$ en Arkansas; $77 \%$ en Nevada; $73 \%$, en Carolina del Norte; 70 \%, en Georgia y $70 \%$, en 
Nebraska (Edwing García y Ben Stocking, Latinos on the Move to a New Promised Land. San Jose Mercury News, 16 de agosto de 1998.).

\subsection{El caso de Wichita, Kansas}

Cuando nos fuimos de Kansas de arrieros, mi "caporal" gritó: “Cuida bien a mi amor!".

Después llegan cinco mexicanos, todos con buenas chaparreras;

$y$ en menos de un cuarto de hora, tienen todas las reses acorraladas.

$Y$ luego se pudo ver Kansas, y el "caporal" nos dice:

"Lo logramos, pronto las tendremos en el corral".

Ya con esta me despido, por el amor de mi muchacha;

canté para mis amigos el corrido del arriero'

En Kansas, el número de latinos en el condado de Sedgwick (Wichita), creció en un $35 \%$-de 17,611 personas a 23,866-, entre 1990 a 1996. En otros condados de Kansas, la población mexicana está creciendo del 35 al $55 \%$. La gran mayoría de los latinos recién llegados son residentes legales mexicanos que, gradualmente, se dirigen a Wichita en busca de trabajo. La tasa de desempleo en Wichita se mantiene por debajo del $3.5 \%$. En todo el estado, la población latina ha crecido en más de $32 \%$-de 94,241 a 124, 842 - a partir de 1990. Esto representa aproximadamente el $5.5 \%$ de la población estatal, comparado con cerca del $11 \%$ de la población nacional. En los pueblos más pequeños, encuentran trabajo en granjas y en plantas empacadoras, mientras que en las ciudades más grandes lo hacen en la construcción y los servicios. De hecho, si prevalece la tendencia actual, los latinos se convertirán en el grupo minoritario más grande en menos de cinco años. Actualmente, los afroamericanos son el grupo mayor, estimado en 152,166 personas. En contraste con ello, la población blanca del estado se estima en 2.3 millones. En Wichita, la creciente influencia mexicana está llevando a muchos negocios a proveer servicios hispanoparlantes en las secciones de productos mexicanos. Basta dar un vistazo a la Calle 21, justo al oeste de Broadway, en Wichita, para encontrar banderas mexicanas, murales y anuncios de comida, ropa y tiendas de vídeo mexicanas. La mayor concentración se encuentra en un área de 8.5 kilómetros cuadrados al norte del centro de

1. El corrido Kiansis habla del famoso arreo de ganado mexicano de Texas hacia Kansas en los últimos años de la década de 1860 y los primeros del decenio siguientc. (Cfr. Américo Parcdes, A Texas-Mcxican Cancionero, Urbana, Univcrsity of Illinois Press, 1976.)

La latinización de los Estados Unidos: Inmigración, cultura y transformación 
Wichita, que delimita el oeste de Broadway hacia el río Little Arkansas, cerca de un área industrial.

Muchos latinos vienen a Wichita a competir por empleos en los que la mayoría de los habitantes originarios de la ciudad no están interesados. Aceptan este tipo de trabajos ante la incapacidad de los empleadores para atraer a los nativos de Kansas, quienes saben que pueden conseguir mejores oportunidades en otros lugares.

Las escuelas, las iglesias y otras instituciones están resintiendo los efectos de esta comunidad en ascenso. La inscripción de latinos a los distritos escolares de Wichita se incrementó en más de $45 \%$, en un período de cinco años. Pueden observarse señalizaciones bilingües en las escuelas públicas. Debido a la alta demanda, en 1996, la iglesia católica de Nuestra Señora del Auxilio Perpetuo empezó a ofrecer una segunda misa en español. Muchos mexicanos que salieron de California, debido a la aprobación de la Propuesta 187, en 1994, que le negaría a los trabajadores indocumentados los servicios de salud, bienestar social y educación pública. En 1995, un juez federal decretó que la iniciativa era ilegal, porque las leyes federales la sobreseían. Los sentimientos y actitudes antimigratorias en California hicieron que muchos se fueran a Kansas. Además, Kansas es un lugar más seguro para la educación de los niños y tiene índices de criminalidad menos elevados que California. Eduardo Sebastián afirma que "no hay punto de comparación entre las gloriosas montañas y costas de California y la aburrida planicie del medio oeste, pero cuando se trata de educar adolescentes, el lugar propicio es Kansas" (Joe Rodríguez y Hurst Laviana, More Hispanics Call City Home. The Wichita Eagle, 22 de marzo de 1998.).

\subsection{El caso de Siler City, Carolina del Norte}

En poco más de ocho años, la población latina de esta ciudad ha crecido, de virtualmente cero a 3,200 habitantes — casi $40 \%$ de la población total-. Los datos más recientes estiman que al menos $20 \%$ de estos latinos provienen de California. Muchos se sienten solos por estar lejos de su casa, ya sea México o California. Otros terminan en distritos donde las rentas son bajas, y frecuentemente habitan viviendas por debajo de los estándares de calidad. Se han establecido zonas comerciales con tiendas como la Gabrieh La Popular, la Guerrero, bodegas donde las familias mexicanas se abastecen de tortillas, veladoras, botas... Una agencia aseguradora en Siler City puso el letrero "aseguranzas"2 en

2. Aseguranzas: Palabra en espánglish. Viene del inglés Insurances, seguros de vida. (N. del E.) 
la ventana, en anunciando sus servicios a los clientes latinos. Hay misas en español. Las tiendas tienen letreros en ese mismo idioma. Cuando eran recién llegados, los mexicanos se pusieron a trabajar en granjas avícolas locales. Hoy en día trabajan en virtualmente toda la industria del área: de la construcción hasta la textil. La fábrica de textiles Charles Craft está compuesta ahora en un $760 \%$ por latinos, mientras que hace tan sólo cinco años, la mayoría de los trabajadores eran afroamericanos y blancos. El trabajo ahí es monótono: cambiar y ajustar cientos de carretes de hilo industrial. Las máquinas hiladoras rugen con un estruendo ensordecedor, y el aire está lleno de polvo de algodón. Muchos trabajadores latinos viven justo del otro lado de la fábrica, en una vieja zona de casas pequeñas. No hace mucho, el vecindario estaba compuesto prácticamente en su totalidad por blancos; ahora, en casi cada una de las casas vive un latino. Algunos tienen dificultad en adaptarse a la vida en la Carolina del Norte rural. Desde la época de la esclavitud, la ciudad estaba compuesta por afroamericanos y blancos. Del total de personas que aprende inglés, el $25 \%$ lo habla con acento mexicano; el $75 \%$ restante, lo hace con acento sureño. Ha habido algunos matrimonios entre blancos y latinos. Muchos de los que emigran a Siler City son atraídos por el bajísimo costo de la vida. En esta área, la gente puede rentar o comprar casas, mientras hacerlo en California sería imposible.

En Carolina del Norte, se han agudizado las tensiones de manera especial entre los latinos y los afroamericanos, quienes componen el $22 \%$ de la población del estado. Los latinos se han mudado a los barrios negros en busca de arrendamientos menos caros. Los afroamericanos ven a los latinos como rivales en la lucha por el empleo y la vivienda. Ellos solían predominar en muchos lugares de trabajo cuyo único sostén es hoy el trabajo latino. Los afroamericanos constituyen el $25 \%$ de la población en Soler City. "Se quedan con los empleos estadounidenses y no pagan impuestos", ha afirmado un residente afroamericano. Hay tantos mexicanos de California en Siler City, que uno de los residentes sugirió que se cambiase el nombre de la ciudad al de "Nueva California". (Ben Stocking y Edwin García, Middle America Grapples With An Influx of Latinos. San Jose Mercury News, 17 de agosto de 1998.)

\subsection{El caso de Nueva York}

Los mexicanos han emergido como uno de los grupos inmigrantes más recientes y con un crecimiento más veloz, en Nueva York, especialmente a partir de 1990. Comenzaron a emigrar a esa ciudad hacia la Segunda Guerra Mundial, siguiendo un patrón de migración en cadena, que hizo explo-

La latinización de los Estados Unidos: Inmigración, cultura y transformación 
sión en los últimos años de la década de los ochenta. El censo de 1990 registró 61,722 personas que declararon ser de origen mexicano, en contraposición a las 22,577 registradas en 1980 . Otras fuentes estimaron el número en cerca de 100,000 personas para 1990. En la actualidad, hay aproximadamente 230,000 mexicanos en la ciudad, junto a otros 100,000 que viven en el área metropolitana. Los mexicanos son el grupo inmigrante que crece a mayor velocidad en la urbe. Son el cuarto grupo inmigrante más grande de la ciudad, después de los dominicanos, los chinos y los jamaiquinos.

Una característica distintiva de la población mexicana en Nueva York es su falta de concentración en un área particular. Muchos viven en Brooklin, algunos menos en Staten Island, pero también es posible encontrarlos en Queens, Manhattan y el Bronx. ¿Qué empleos tienen los mexicanos? La mayoría trabaja en restaurantes y en servicios (como trabajadores domésticos, mensajeros y abarroteros), así como en fábricas. Trabajan más horas por un salario menor.

Casi cada historia migratoria es el drama de una familia, sus aspiraciones y frustraciones, sus separaciones y reencuentros, sus tradiciones y compromisos. Para una familia mexicana del estado de Puebla que ahora vive en Nueva York, es también la historia de un pueblo sin fronteras, una aldea global, en el sentido más literal de la expresión: Un pueblo mexicano que trasciende toda fronteras. Con tarifas aéreas bajas, tarjetas telefónicas internacionales, la videocasetera $y$, ahora, con una ley que permite a los mexicanos conservar su nacionalidad original mientras son ciudadanos norteamericanos. Los habitantes de ese pueblo sin fronteras viven entre aqui y allá. Es un grupo inmigrante que se mantiene intacto, al tiempo que se asimila a la vida neoyorquina. Han negociado una doble vida, basada en su intimidad comunitaria. Desde una pequeña aldea del estado de Puebla, se ha gestado la saga de múltiples generaciones de sus habitantes en edad laboral, quienes han emigrado a Nueva York. El pueblo en México se ha mantenido vivo, gracias a los emigrantes que consideran al lugar donde se está como un estado mental, como un asunto del corazón y de raíces espiritual.

En Nueva York, los emigrantes de la primera generación son meseros, trabajadores de la industria del vestido y mecánicos de autobuses. Pero, de vuelta a casa, se transforman en personajes muy importantes, porque han ahorrado dinero que ayudará a construir escuelas, así como a reparar la iglesia; ellos financian y diseñan el sistema de agua potable, y también iluminan las calles. Sus hijos son enviados a la escuela. La segunda y la tercera generaciones se mantienen leales a su pequeño pueblo en México. Muchos mantienen rela- 
ciones con sus hijos al regresar a México cuando dejan de trabajar. Una vez en ese país, montan negocios y construyen casas con sus ahorros.

Muchos regresan a México durante las fiestas navideñas. Pero éstas continúan en Nueva York a lo largo del año. Hay nueve ligas mexicanas de fútbol soccer $^{3}$ en la ciudad, integradas por 262 equipos. Han creado círculos sociales poblanos en Nueva York: Usan las instalaciones de una iglesia o de una tienda de abarrotes. Han integrado comités que generan ingresos para proyectos específicos en su lugar de origen. Los méxico-neoyorquinos envían alrededor de dos millones de dólares a México anualmente. El total enviado desde los Estados Unidos a México en 1997 fue de aproximadamente cinco mil millones de dólares, cifra cercana a los ingresos obtenidos por la producción petrolera y el turismo en este último país.

Los mexicanos nacidos en Nueva York no tienen conflicto alguno acerca de sus identidades. Saben que su arraigo a México es saludable, que enriquece su identidad y que produce un noble sentido comunitario en la ciudad. Pero existe cierta tensión. Muchos jóvenes saben que sería difícil permanecer en un pueblo mexicano pequeño, en una sociedad conservadora, donde, por ejemplo, es mal visto que una mujer fume. No tienen que escoger entre aqui y allá. Siempre tendrán un hogar en México y Nueva York: el privilegio de cambiar entre dos hogares, entre dos mundos (Deborah Sontag, $A$ Mexican Town That Trascends All Borders. New York Times, 21 de julio de 1998; y Robert C. Smith, Mexicans in New York: Membership and Incorporation in a New Inmigrant Community, en Gabriel Haslip-Viera y Sherrie L. Baver (eds.), Latinos in New York, Notre Dame, University of Notre Dame Press, 1996).

Se ha afirmado mucho que los Estados Unidos son una nación de inmigrantes: "una nación de naciones". Por lo tanto, necesitamos repensar la historia estadounidense y el reciente oleaje de inmigración latina que ha repoblado esta nación. Necesitamos centrarnos en los inmigrantes mismos y en las circunstancias históricas que originaron este perfil inmigrante latino tan diverso. La inmigración latina contribuye a crear la nación estadounidense y su historia nos ayuda a comprender mejor el "caleidoscopio" de vida en los Estados Unidos.

3. En los Estados Unidos, para referirsc al fútbol tal como lo conoccmos en Latinoamérica, se emplea el vocablo soccer, para distinguirlo del football, o fútbol americano. ( $N$. (lel E.)

Lo latinización de los Estados Unidos: Inmigrición, culturo y transformación 